\title{
Role of Artificial Intelligence in Distinct Sector: A Study
}

\author{
Harsh Jindal ${ }^{1}$, Devanshu Kumar ${ }^{2}$, Ishika ${ }^{3}$, Santosh Kumar ${ }^{4}$ and Rakesh Kumar ${ }^{5}$ \\ ${ }^{1,2 \& 3}$ Department of Computer Science Engineering, Chandigarh Group of Colleges, Mohali, Punjab, India \\ ${ }^{4 \& 5}$ Department of Mechanical Engineering, Chandigarh Group of College, Mohali, Punjab, India \\ ${ }^{4} \mathrm{PhD}$ Research Scholar, Department of Mechanical Engineering, IKG Punjab Technical University Kapurthala, Punjab, India \\ ${ }^{5} \mathrm{PhD}$ Research Scholar, Department of Mechanical Engineering, Chandigarh University, Mohali, Punjab, India \\ E-mail: rk05691@gmail.com
}

\begin{abstract}
The artificial intelligence (AI) plays a significant role in distinct fields to solve the complex problems. The digital technical field is increasing day by day in the world and it makes an internal part of our life. Hence, the knowledge of emerging technology is must for making our life easy. However, there are some major areas which are creating the problems to human such as agriculture field that comprises crop diseases, lack of storage management, pesticide control etc. These problems can be solved by artificial intelligence, IOT, machine learning and deep learning. Hence, the aim of this paper is to discuss the role of artificial intelligence to solve different issues of distinct sectors (medical, engineering, agriculture, business, defenses etc.) especially in medicine (COVID-19). Finally, future scope, challenges and application domain of artificial intelligence is also described.

Keywords: Artificial Intelligence, Applications, Benefits, Future Scope, Challenges
\end{abstract}

\section{INTRODUCTION}

AI is the ability of a digital computer or a robot to perform tasks commonly associated with intelligent beings. The term is frequently applied to the project of developing systems endowed with the intellectual processes characteristic of humans, such as the ability to reason, discover meaning, generalize, or learn from past experience. Since the development of the digital computer in the 1940s, it has been demonstrated that computers can be programmed to carry out very complex tasks-as, for example, discovering proofs for mathematical theorems or playing chess-with great proficiency.

Artificial Intelligence (AI) is one of the emerging technologies in the distinct industrial sector. It is utilized for the processing of visual information to symbolic reasoning, task planning, self-learning and recognizing actions, etc. The forecasting behavior has been made economical, and faster with introduction of distinct $\mathrm{Al}$ components [1]. We need to concede that computerized advances have effectively become an indoor a piece of our way of life. With the appearance of innovation during this computerized world, our people have stretched our boundary of the reasoning cycle and attempt to combine typical cerebrum with a man-made one. Knowledge is coordinated with different psychological capacities, for example, language, consideration, arranging, memory, discernment. The development of knowledge can essentially be concentrated about in the most recent ten years. Insight includes both Human and Artificial Intelligence. Man-made brainpower was brought into the world in close association with the executives' science, grew apart from it, and is currently framing new connections with it, just as with different controls that have met up in cognitive science. Numerous new innovations were brought into the world in this always expanding innovation field like Artificial Intelligence. Computerized reasoning is a simple interaction: with the assistance of which human can make a machine working like a human psyche. Man-made intelligence has infiltrated in life science, instructional, accountability, agribusiness, industry, security, and loads of different areas. Execution of AI includes learning cycle of machines. As of late an ever increasing number of various intelligent instructional innovations are getting famous. Man-made consciousness is that movement dedicated to making machines clever, and knowledge is that quality that empowers a substance to work fittingly and with premonition in its current circumstance". As per report, "the capacity to utilize AI to support choosing, rethink plans of action and biological systems, and revamp the client experience will drive the result of computerized activities through 2025". They additionally showed that $59 \%$ of associations are probably going to execute AI techniques, just as others have effectively gained ground in directing or embracing AI arrangements. That could be referenced various areas including schooling [1].

\section{A. Artificial Intelligence}

Artificial intelligence in computer science, engineering refers to any human like intelligence provided by a robot or computer or machine. The major applications of AI refer to the ability of a machine or computer to mimic the capabilities of the human mind learning from experience $\&$ examples, understanding and responding to language, making decisions, problem solving, etc. We, as a whole will in general utilize a typical meaning of computerized reasoning when we have got some information about it. What is man-made brainpower? We call a program for a PC "misleadingly keen" in the event that it accomplishes something which, when done by an individual, will be thought to require human insight. We characterize it regarding the undertakings that are done. We thought we were utilizing our insight, yet these days, maybe $90 \%$ of the 
exertion of PCs is given to mathematical investigation and we don't consider that counterfeit intelligence.AI is a device which like arithmetic or, besides, similar to language, spreads through the entire society. Everyone needs to utilize this device and numerous individuals will be imaginative in its utilization, not simply individuals who extravagant themselves to be experts. Not all the writing on the planet is finished by expert/teachers of Chinese or English or of any common language [2]. So we are in that impossible to miss position where a specific subset of the clever things that PCs do are singled out and called man-made consciousness and the limits are actually very subjective. In the previous 3 or 4 years-possibly somewhat more than that, yet since the time that man-made brainpower has started to get a ton of public consideration-I have detected a drawing together again of these fields. So progressively again today, and I think it is an awesome turn of events, we are starting to see man-made reasoning abilities being united with conventional administration science and activities research abilities.

\section{B. Why AI is most Demanding?}

Man-made intelligence apparatuses exist for quite a long time, however, over the most recent few years; its appropriation has expanded to a more significant level. There exist numerous reasons that portray the significance of AI models and make them more adorable. These reasons are: [3].

1. Calculation Power and Cost: Using AI strategies and calculations, the calculation power in business activities can arrive at a significant level. With a mix of the Internet of Things (IoT), AI creates an immense volume of information that makes the AI more effective.

2. Age of Huge Datasets: Using big data and its innovations, tremendous datasets are created. The more the information will be, the more productive the AI-model will be the part of the open development in AI relates to the simple admittance to AI-based abilities and thinking. Thusly, more individuals will be exploiting AI development and ll the hole between these innovations and people. People building up the AI-models are considering the quick and dirty subtleties, sending Neural organizations and produce significant level models that gain proficiency with every second and perform activities true to form. Other than wars, artificial insight is completely trusted and consequently being the effective accomplishments in different businesses and use-cases, the human upset indeed ventures up in advance.

The influence of artificial knowledge on living souls as well as economy has been surprising. Man-made consciousness can add about \$15.7 trillion to the world economy by 2030 . To bring that into a view point that about the consolidated financial yield of china and India starting today. With different organizations foreseeing that the utilization of AL can support business efficiency by up to $40 \%$, the emotional expansion in the quantity of AL start-up has amplified multiple times since 2000 the use of AL can go from following space rocks and other inestimable bodies in space to anticipate sicknesses on earth, investigate new and imaginative approaches to control psychological oppression to make mechanical plans[4].In addition, the major challenges in artificial intelligence are represented in Figure 1.

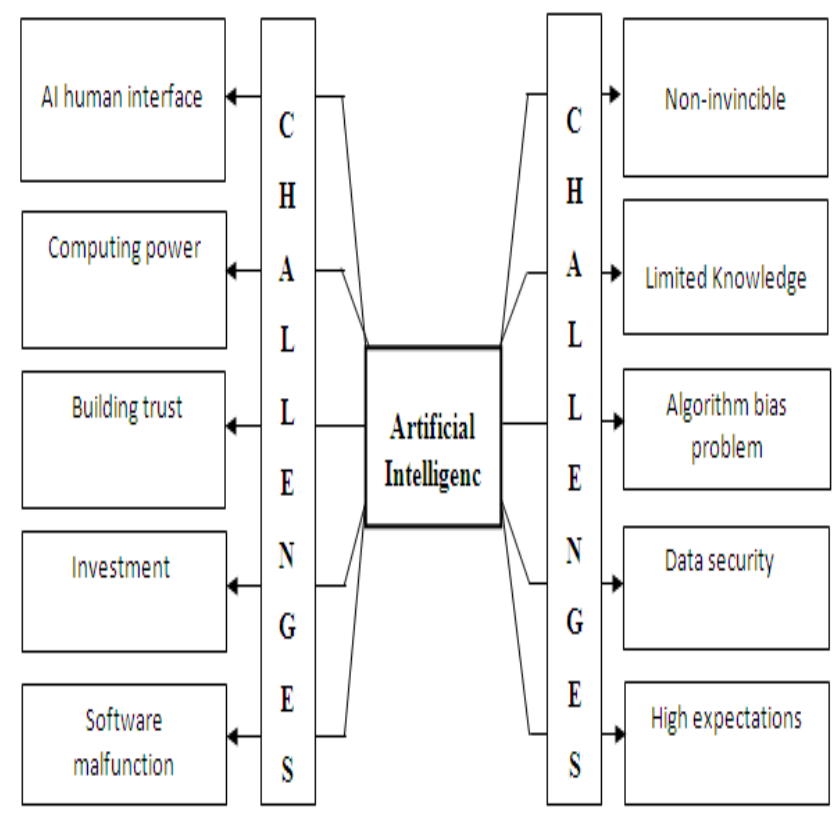

Fig. 1 Challenges in artificial intelligence [4]

3. Computing Power: The measure of force these calculations use is a factor that holds numerous designers back from moving. AI and top to bottom learning are the stepping stones of this computerized reasoning, and they require a developing absence of controls and GPUs for ideal execution. There are different fields where we have the thoughts and information to use top to bottom learning structures like space rock following, wellbeing sending, enormous body following and considerably more. They need a PC registering force and indeed, huge PCs are reasonable. Albeit because of the accessibility of distributed computing and comparable preparing frameworks they work in AL frameworks with incredible achievement include some significant downfalls.

4. Limited Knowledge: In spite of the fact that there are numerous spots in the market where we can utilize Artificial Intelligence as a superior option in contrast to conventional frameworks. The genuine issue is the data on Artificial Intelligence. Beside innovation sweethearts, understudies, and specialists, just a small bunch of individuals know the force of AI. For instance, there are numerous SMEs who can get their work coordinated or learn better approaches to develop their item, oversee assets, sell and oversee items on the web, learn and comprehend purchasers conduct and react to the market successfully and proficiently. They don't have the foggiest idea about any specialist organizations 
like Google Cloud, Amazon Web Services, and others in the innovation business.

5. Human Level: This is perhaps the main difficulties for AI, which has kept scientists at the edge of AI administrations in organizations and new businesses. These organizations may flaunt over $90 \%$ exactness; however individuals can improve taking all things together of these circumstances. For instance, let our model foresee whether the picture is a canine or a feline. One can foresee the right yield quite often, killing a shocking precision of over $99 \%$. For a top to bottom learning model to play out a similar assignment would require extraordinary financing, the utilization of a hyper parameter, an enormous data set, and an all-around characterized and precise calculation, just as amazing PC power, continuous preparing in train information and test information testing. That seems as though a great deal of work, and it's in reality multiple times harder than it sounds. Another approach to try not to do all the difficult work is to utilize a specialist organization, as they can prepare top to bottom learning models utilizing pre-prepared models. They are prepared in a huge number of photos and are efficient with high exactness, yet the genuine issue is that they keep on showing blemishes and will battle hard to arrive at human execution.

6. Data Privacy and Security: A vital factor on which all top to bottom and mechanical learning models are based is information obtaining and preparing assets. Indeed, we do have information, yet since this information is created from a huge number of clients around the world, there is likelihood that this information might be utilized for noxious purposes. For instance, assume a medical care supplier offers types of assistance to 1 million individuals in a city, and because of digital assaults, the individual subtleties of each 1,000,000 clients fall under the control of everybody on the dark web. This information remembers information for sicknesses, medical issues, clinical history, and the sky is the limit from there. To exacerbate the situation, we are presently managing planet size information. With such a lot of approaching data coming from all pointers, there will unquestionably be a few instances of information spill. A few organizations have started to work more brilliant than any time in recent memory. It trains information on brilliant gadgets, so it isn't getting back to the workers; just a prepared model is gotten back to the association.

7. Bias Problem: The positive or negative of an AI framework relies to a great extent upon the measure of information they are prepared to. In this manner, the capacity to get great information is an answer for great AI programs later on. However, the everyday information gathering associations is undesirable and has no worth. They are one-sided, and by one way or another characterize the nature and the meaning of a set number of individuals with comparable interests as far as religion, race, sex, local area, and other racial biases. Genuine change must be achieved by characterizing different calculations that can more readily follow these issues.

\section{SCOPE OF ARTIFICIAL INTELLIGENCE TO HANDLE COVID-19}

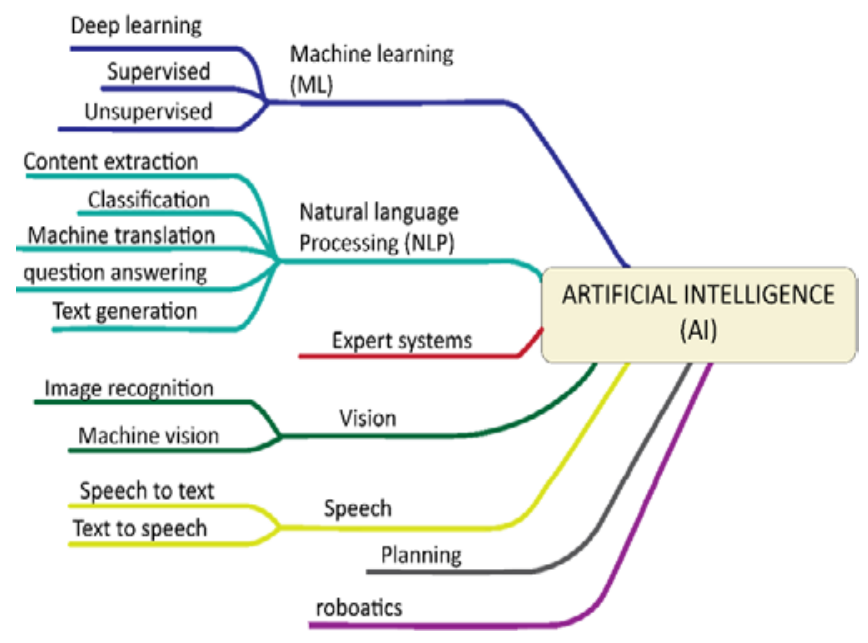

Fig. 2 Branches of artificial intelligence. [5]

\section{A. AI based Model for Treatment of COVID-19 Patient}

The AI-Based model should be created to battle against the outstanding climb of individuals getting contaminated with the Covid .Patients having manifestations of hack and fever should be screened and in the event that they are tried positive should keep confinement and isolate for 14 days. Acquire the amount of the tainted individual. Geotagging is utilized for contact following of a COVID-19 patient includes "Infection investigators" ground level human specialists who go house to house following the developments of positive patients other than utilizing CCTV for signs or ID framework to validate severe recognition of home isolate. After surpassing the amount of isolating and tried positive, medicines are visiting be given to the patient and screened again to check whether the patient is recuperating [6].

\section{B. AI Based Fever Detection System}

The Athena Fever Detection System gives snappy, right mass evaluating for raising temperatures. The framework is non-obtrusive, non-contact, and quick. The framework distinguishes the essence of the subject, disregards problem areas like hot lights above and other hot items on the individual like a wireless or hot espresso. The individual ganders at the camera, and the framework finds the most blazing points on the face close to the eyes, called the internal canthus. Athena is most widely utilized as the $1^{\text {st }}$ of a two designated spot system where fever is then affirmed by a few second clinical thermometer. The temperature estimation is started at differing areas around utilizing infrared imaging framework and handheld thermometers. The far off fever location framework is acclimated with 
measure a human temperature in a very group a good ways off of up to 5 meters [7].

\section{AI for Virus Detection and Epidemic Prediction}

Man-made brainpower is by and large, wide used in the circle of medication to prevent, control, and predicts the spread of infections. With new irruptions like the current episode of the Covid, AI is making the principal sway. We realize that even before the information on the Corona infection irruption opened up to the world, a Canadianbased figuring (AI) based organization; Blue Dot previously had raised partner early alert. Artificial Intelligence has returned an all-inclusive way in plague forecast and understanding it's examples. Not exclusively that, by investigating the overall carrier tagging information, the corporate furthermore expected that the ailment may unfurl to places like capital of Japan, Bangkok, Seoul and Taipei Blue Dot utilize phonetic correspondence interaction and AI to winnow information from a huge number of sources, just as articulations from true general wellbeing associations, advanced media, and world carrier it's prepared to apace technique loads of information each quarter-hour, 24 hours day by day [8].

\section{Al based Patient Screening System}

Evaluating an outsized scope of suspected patients for adequate isolate and treatment might be a need task that needs a fast and right indicative method to manage the outspread of (COVID-19). Pathogenic lab testing is long with significant false negative outcomes. Registering is Associate in a nursing relationship of profound discovering that might be utilized which may decide tomography changes in CT pictures. The profound learning procedure will extricate COVID19's graphical choices to create a clinical assignment past the infective investigate so saving significant time for wellness the board A framework projected to utilize solid 2D and 3D profound learning models, altering and adjusting existing AI models and blending them in with the clinical arrangement [9].

\section{E. Robots and Drones Providing Health Care Services to COVID Patients}

Medical services specialists are compelled to pioneer and utilize innovation to battle the outspread of pandemic along the edge of conveying quick and safe therapy. However, there are three zones drones are regularly used to help from enormous scourge transferable infection.

1. The elevated perception huge scourge infectious illnesses unfurl size assessment.

2. The enormous scourge infectious sicknesses legitimate and item conveyance.

3. The post enormous pandemic infectious sicknesses investigation.
Reconnaissance drones are adjusted to screen people all through lockdowns, scattering public get-together, traffic perception, garbage removal perception, decides people not after friendly separating and not utilizing the cover and decide those that have overstepped the country's restriction laws. Robots are utilized to fly clinical examples and isolate materials all through the outspread of adjacent contamination COVID-19 [10].

\section{F. Rise of AI makes Emotional Intelligence more Important}

There are simply so numerous things machines can show improvement over people, and we ought not to be too pleased to even consider conceding them. Numerous talented positions follow a similar general progression of work: (i) Gather information (ii) Dissect information (iii) Decipher the outcomes (iv) Choose the suggested strategy (v) Start the activity We can take a gander at quite a few errands to check whether this end is valid. Generally the doctors perform tests, plan a treatment, and afterward activity with the patient to perform the treatment reasonably. Monetary consultants gather and break down data about their customers and possible vehicles to contribute, decipher results acquired from an assortment of components, for example, hazard resistance, suggest a speculation methodology, and assist their customers with building up the system over the long run. Business consultants do likewise, however, analyze and tackle business issues. These profoundly talented specialists can handle excessive costs due to the three abilities. Their capacity to traverse the underlying catch exercises rapidly and precisely; their experience and judgment in deciding the strategy; and their capacity to assist customers with exploring that course. Simulated intelligence and AI will rapidly outperform our abilities in two abilities - and this will change the abilities required for any worker who wishes to remain in these positions as they are changed by imaginative resourcefulness [11].

It is not difficult to see the part of computerized frameworks in information assortment and investigation. We acknowledge that machines can do these sorts of occupations well. A few group may say we can never believe machines with significant choices like the administration of our lives and cash, yet this is a 20th century thinking. In any case, the new age is offering to its brilliant, trusted, and regularly modern gear. Besides, it is hard for anybody to contend with the outcomes. Regardless, these abilities will be fundamental for any individual who needs to remain important in their field as robotized programs multiply. We have three proposals: Do not battle for mechanical progression. AI and AI can possibly improve results and lower costs - so don't battle robots. Embrace change in your industry and work to make it more gainful and predictable. Test your relational abilities, propel and test individuals. Know about your qualities and shortcomings with regards to passionate knowledge. Invest in improving your passionate insight. A simple method to change your psychological model about what's significant in 
your job, and afterward begin zeroing in on how you can more readily oversee, impact and identify with others.

\section{FUTURE SCOPE OF ARTIFICIAL INTELLIGENCE}

\section{A. Artificial Science in Science and Research}

Man-made reasoning has a decent extension in enhancing life, and the numbers have been demonstrating this case from that point forward. For instance, in 2019, there was a discovery in medication and AI when scientists discovered that the beginning of bosom disease can be identified precisely almost immediately and can assist specialists with distinguishing terms of favourable and threatening cells. This is only one model among numerous while considering the future extent of man-made brainpower to comprehend that AI can be put to viable use in any field. An extraordinary model is 'Eve,' which is an AI-based robot. It found an element of toothpaste that can fix a hazardous sickness like Malaria. Envision a typical substance present in an ordinary thing that is fit for treating Malaria. Biotechnology is another field where analysts are utilizing AI to plan microorganisms for mechanical applications [12]. Thinking about the entirety of this, it is protected to assume that AI will quicken logical examination and application quickly.

\section{B. Artificial Intelligence in Agriculture}

The world necessities to create more than $50 \%$ of its present produce in simply one more 30 years' time. Cautious arranging of assets close by AI will assist ranchers with developing maintainable products while overseeing assets in an astounding way. Considering elements, for example, environmental change and populace development, AI can possibly give inventive answers for improve the harvest yield over time to fulfil the rising needs. The future extent of man-made consciousness includes utilizing robots to help in crop wellbeing recognition, collecting and soil investigation. This will without a doubt guarantee positive outcomes in the long haul. A basic apparatus, for example, a cell phone camera can be utilized to play out a wide assortment of examination and tests in the field of horticulture. In the event that this is combined with AI, the prospects are basically unending with gigantic potential [13].

\section{Artificial Intelligence in Cyber Security}

We face a daily reality, such that everybody is worried for the well being of their information and their security. This has been a drawn out worry for a long time and network protection was the response to the issue. The principal thing that comes into mind when there is any discussion about extortion is the Visa tricks that have been going on for the most recent few years. To keep their information and assets secure, associations are making gigantic interests in network protection. The future extent of AI in network safety is splendid. Numerous organizations are utilizing AI-based answers for computerizing the dull cycles present in online protection. For instance, IBM has IBM resilient, which is a rationalist and open stage that gives the foundation and the center point for overseeing security reactions. Another field is misrepresentation discovery. Simulated intelligence can help in distinguishing fakes and help associations and individuals in keeping away from tricks. For instance, repetitive neural network fit for identifying misrepresentation in their beginning phases. They can examine broad amounts of exchanges rapidly and characterize them as per their dependability [14].

\section{AI in Data Analysis}

Information is the new oil and Artificial Intelligence is the new motor. At the point when the information has achieved a particularly incredible status in this day and age where it is leading a few organizations to progress and contriving sharp and succinct answers to the world's issues, it would profit enormously if the force of AI is utilized to distinguish examples and patterns that are not noticeable to the natural eye. Computer based intelligence can distinguish examples and bits of knowledge that natural eyes can't see without investing in a great deal of energy. Additionally, it is quicker and more adaptable at doing as such. You can ask Analytics Intelligence an inquiry in straightforward English, and it would give you a brief answer. It additionally furnishes website admins with Smart Lists, Smart Goals, Conversion Probability, and different highlights that help the website admin in improving the after effects of their webpage. The extent of AI in information examination is rising quickly [15].

\section{E. Al in Power Station}

Thermal power plants, nuclear power plants and hydro power plants are three distinct categories of power plants for electricity generation. The $\mathrm{Al}$ plays major role in power systems applications that includes to enhance the efficiency of boiler parts, control of power (Power flow control, voltage control etc.), The distribution system (network reconfiguration, operation \& planning, demand side management etc.), Power system planning (power system reliability, reactive power planning etc.), Forecasting (long \& short, solar \& wind power forecasting etc.), strategy games and control of network, etc. [A].

In addition, the major problems in thermal power plant are corrosion, oxidation and erosion. However, various authors [16-29] have used various types of coatings to combat high temperature oxidation, erosion corrosion. But, the use of AL in power plant can play a significant role. The three distinct approaches of $\mathrm{Al}$ and their features comparison in power station protection is illustrated in Figure 3. 


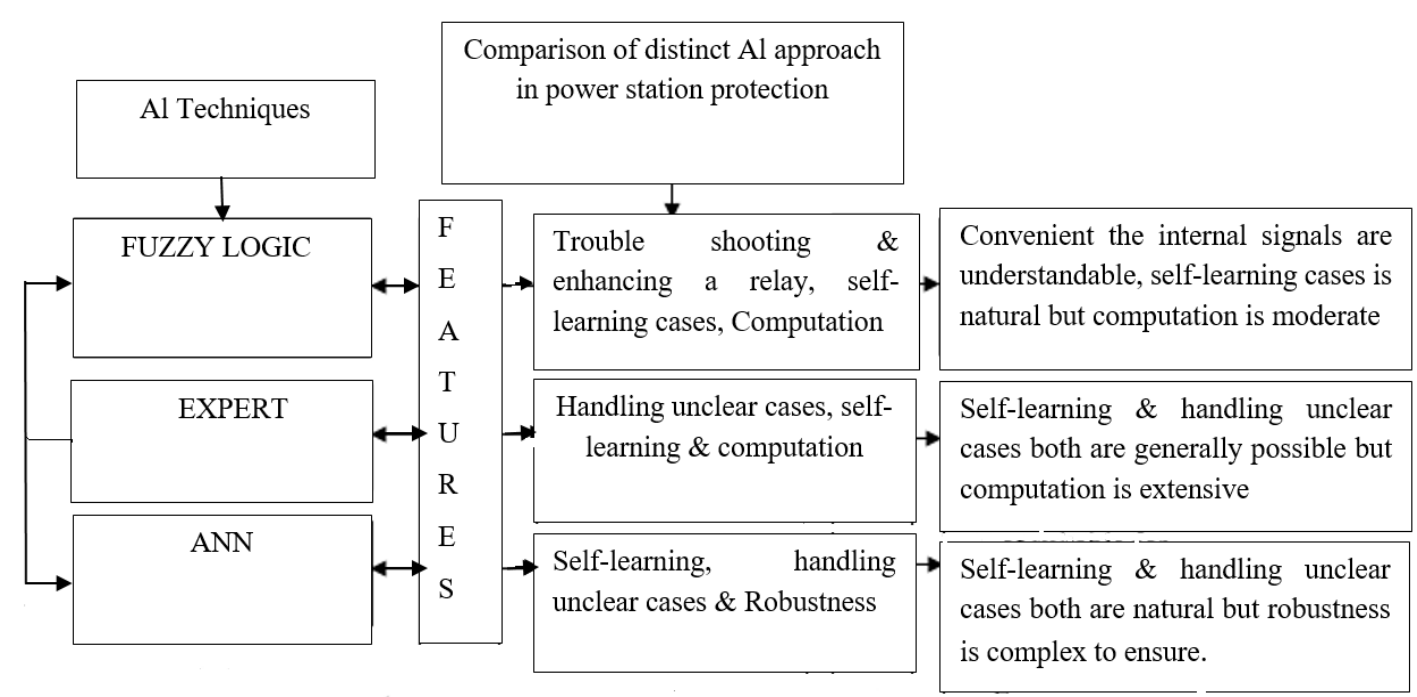

Fig. 3 Comparison of three distinct Al techniques and their feature in power system protection [30]

\section{F. Simulated Intelligence in Healthcare}

The clinical area is likewise utilizing this innovation for its benefits. Computer based intelligence is helping clinical analysts and experts from various perspectives. We've effectively referenced how AI is helping scientists in their field as well. Aside from finding a remedy for disease, a few associations are utilizing AI to assist patients with getting telemedicine. The UK's National Health Service utilizes Google's Deep Mind stage to identify well-being chances in individuals through applications. Simulated intelligence can assist specialists in keeping away from these mistakes by furnishing them with applicable data sets and proposals. It can break down the data set of patients with comparative side effects and recommend the treatment that was the best in those cases. Many significant associations, including IBM and Microsoft, are working together with clinical establishments to take care of the different issues present in the medical care area [31]. The distinct applications of M.L. kits and M.L. models in medical affairs are shown in Figure 4.

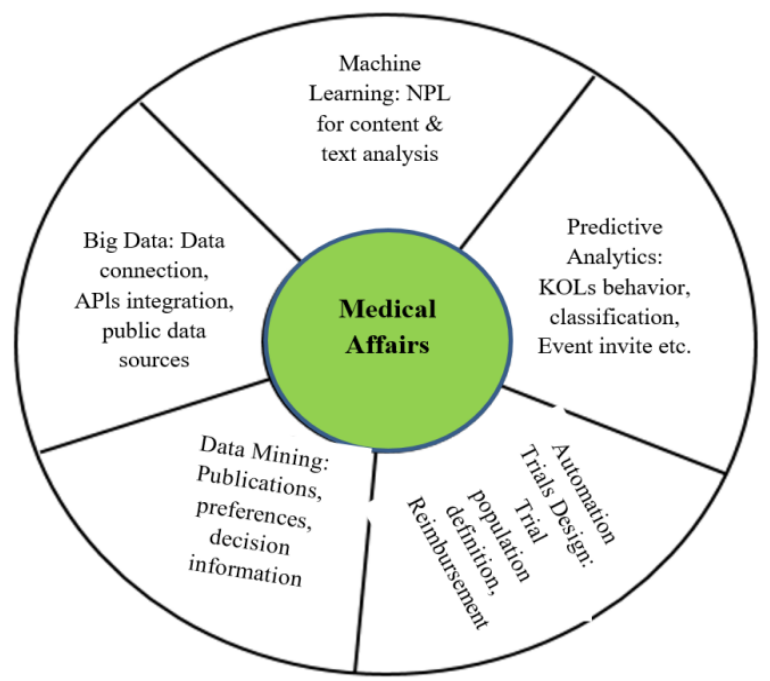

Fig. 4 Use of M.L. kits and M.L. models in medical affairs [32]

\section{G. Market Value}

The "Man-made brainpower Market in Homeland Security, Public Safety and National Security: 2020-2025" report conjectures that this current industry's incomes will develop at a 2019-2025 CAGR of $18.5 \%$. The utilization of AI and AI by country security, protection, public well being associations and insight organizations is on the ascent, for the most part on the grounds that the world is getting more computerized and associated. This is setting out new open doors, for informative assortment and capacity, yet in addition for insight handling, misuse, dispersal, and examination. Man-made brainpower innovations can expand the insightful abilities of knowledge associations in numerous significant angles, including: battle on wrongdoing and dread, protection from digital assaults, public security examination, calamity and mass occurrence the executives, and advancement of prescient capacities. All fields of Intelligence profit by AI development including Big Data and Data Analytics Market, Osint Market, Sigint Market, Cyber guard exercises, monetary examinations [33]. This market report, covering country security and public safety AI, is a significant asset for chiefs keen on AI, $\mathrm{AI}$ and large information insight markets. It has been expressly redone for likely financial backers, AI organizations, enormous information organizations, and knowledge offices just as security and government leaders, to empower them to benchmark field-tested strategies, just as recognize business openings, arising innovations, market patterns, and dangers. Questions replied in these market reports that consist of; what will be the AI market dimension in country security, public wellbeing and public safety in 2019-2025? What will the pertinent patterns be during that time? Which submarkets in this sector give appealing business openings? Which nations are relied upon to put most of AI abilities inside the country security, public wellbeing and public safety associations? Which unmistakable content will be used by country security, public safety offices, public wellbeing which innovations 
will be generally required to misuse them? Which market areas are assessed to utilize computerized reasoning abilities? What are the AI \& AI in country security, public wellbeing and public safety market drivers and inhibitors? The report centers around man-made brainpower spending in the Homeland Security, National security market by investigating 4 major key autonomous viewpoints. Every perspective is investigated altogether to more readily comprehend the market and the business openings it holds, including: 20 public business sectors, 3 mechanical business sectors, 4 innovative sub-markets, 8 vertical business sectors. The country security and public safety AI market estimate report present in 450 pages, 85 tables and 205 figures, examination of 19 public, 8 vertical, 7 mechanical business sectors and 9 particular information sources. The report presents for each sub market 2019 information and tasks the 2020-2025 AI market gauge and advances. With a profoundly divided market, we address the "cash trail" every dollar spent - through the accompanying 5 symmetrical market portions: By 19 National AI Markets: USA, Germany, France , Canada, UK, Australia, Italy, India, Spain, China, Japan, Brazil, South Korea, Mexico, GCC, South Africa, Colombia, Kenya, Nigeria. In addition the 7 vertical markets include; Government Intelligence Agencies, Law Enforcement and first Responders, for example, police knowledge, Monetary Services Industry and Fraud Detection: Digital protection Agencies, Boundary Control and Customs, Protection/Military Intelligence Basic Infrastructure Security (counting Mass Transportation Security) and 5 Technological Markets: Picture Analysis, Prescient Analysis, Common Language Processing (NLP), Discourse and Voice Recognition. Other AI Technologies: The AI in country security and public market report presents driving organizations working on the lookout (profiles, items and contact data): that comprises NASA, Cybernet, Amazon, Microsoft etc. Using Artificial Intelligence to dissect and better comprehend the Coronavirus is for the most part being finished by the medical care industry, however not just. Man-made reasoning strategies are appropriate for following, controlling and forecasting the spread of COVID-19 around the globe [34]. Country Security and Intelligence offices are partaking in the battle to moderate
COVID-19 and are using large information, examination and AI to do as such. Geo-area and other cell phone following advances are just of a portion of the AI innovations that are being utilized worldwide by these offices as a component of a bigger procedure of individuals following and following to relieve the pandemic. As per our investigation, there are four distinct methodologies or levels for reconnaissance that legislatures have decided to execute, while utilizing individual information at various levels to monitor their residents. In many nations, country security and public wellbeing organizations are participating in these activities [35].

Germany is utilizing versatile organization information. The cell transporter Deutsche Telekom has imparted area information to the public authority to break down patterns and large information knowledge, not explicit developments. All in all, information from mobile phone transporters was utilized in an amassed, anonym zed configuration to ensure no protection issues were broken. South Korea utilizing Geo-area information. Utilizing Geofinding information, the public authority messages individuals to inform them as to whether they were nearby an analyzed person. The data passed on can incorporate the individual's age, sex, and definite area history. China utilizing Geo-area information alongside extra mass reconnaissance innovations.

The reconnaissance components are the most inescapable and draconian. Notwithstanding the utilization of their mass video reconnaissance (CCTV) frameworks, the public authority likewise uses the far reaching applications Alipay and We Chat. These applications currently incorporate wellbeing codes yellow or green or red set by the Chinese government.

This wellbeing status is then utilized in many urban communities (and soon from one side of the country to the other) to decide if individuals are permitted to ride the metro, take a train, enter a structure, or even leave an expressway. The different AI applications with examples of distinct corona virus stages of the crisis are illustrated in Figure 5.



Fig. 5 AI applications with examples at distinct Corona virus stages of the crisis [36] 
Utilizing PCs to impart is certainly not a groundbreaking thought using any and all means, yet making direct interfaces among innovation and the human brain without the requirement for consoles, mice, and screens is a front line space of examination that has critical applications for certain patients. Neurological illnesses and injury to the sensory system can remove a few patients' capacities to talk, move, and collaborate definitively with individuals and their surroundings. Cerebrum PC interfaces (BCIs) supported by man-made consciousness could re-establish those central encounters with the individuals who dreaded them lost for eternity. "In case was in the nervous system science ICU on a Monday, and I see somebody who has unexpectedly lost the capacity to move or to talk, we need to re-establish that capacity to convey by Tuesday," said Leigh Hochberg, MD, $\mathrm{PhD}$, Director of the Centre for Neuro technology and Neurorecovery at MGH. By utilizing a BCI and man-made consciousness, we can translate the neural initiates related with the planned development of one's hand, and we ought to have the option to permit that individual to convey similar route as numerous individuals in this room have imparted at any rate multiple times throughout the early daytime utilizing a pervasive correspondence innovation like a tablet PC or telephone. Mind PC interfaces could definitely improve personal satisfaction for patients with ALS, strokes, or secured disorder, just as the 500,000 individuals overall who experience spinal string wounds each year [37].

\section{H. Building up the Next Generation of Radiology Tools}

Radiological pictures got by MRI machines, CT scanners, and $\mathrm{x}$-beams offer non-intrusive deceivability into the internal operations of the human body. Be that as it may, numerous demonstrative cycles actually depend on actual tissue tests acquired through biopsies, which convey, chances including the potential for disease. Man-made reasoning will empower the up and coming age of radiological instruments that are exact and itemized enough to substitute the requirement for tissue tests at times, specialists anticipate. We need to unite the analytic imaging group with the specialist or interventional radiologist and the pathologist," said Alexandra Golby, MD, Director of Image-Guided Neurosurgery at Brigham and Women's Hospital (BWH). "That meeting up of various groups and adjusting objectives is a major test." "In the event that we need the imaging to give us data that we by and buy get from tissue tests, at that point we must have the option to accomplish exceptionally close enlistment so the ground truth for some random pixel is known." Prevailing in this mission may permit clinicians to build up a more exact comprehension of how tumors act overall as opposed to putting together treatment choices with respect to the properties of a little portion of the threat [38] Suppliers may likewise have the option to more readily characterize the forcefulness of diseases and target medicines all the more properly. Man-made brainpower is assisting with empowering "virtual biopsies" and advance the inventive field of radio mics, which centers around bridling picture based calculations to portray the aggregates and hereditary properties of tumors [38].

\section{Growing Access to Care in Underserved or Developing Regions}

Deficiencies of prepared medical care suppliers, including ultrasound experts and radiologists can altogether restrict admittance to life-saving consideration in agricultural countries all throughout the planet. More radiologists work in the about six emergency clinics coating the prestigious Longwood Avenue in Boston than in all of West Africa, the meeting called attention to the man-made reasoning could help moderate the effects of this extreme deficiency of qualified clinical staff by assuming control over a portion of the symptomatic obligations ordinarily apportioned to people. For instance, AI imaging instruments can evaluate chest $\mathrm{x}$-beams for indications of tuberculosis, regularly accomplishing a degree of exactness practically identical to people. This ability could be sent through an application accessible to suppliers in low-asset regions, decreasing the requirement for a prepared analytic radiologist on location. "The potential for this tech to expand admittance to medical services is enormous," said Jayashree Kalpathy-Cramer, $\mathrm{PhD}$, Assistant in Neuroscience at $\mathrm{MGH}$ and Associate Professor of Radiology [39].

\section{DISEASES AND IMPACT OF AI ON THEM}

A classic way to repurpose drugs is through network medicine, which includes the construction of medical knowledge graphs containing the relationships between different kinds of medical entities (e.g. diseases, drugs, and proteins) and predicts new links between existing approved drugs and diseases (eg, COVID-19), Methods that are based on graph embedding have been gaining attention for link prediction in graphs that represent nodes and edges as lowdimensional feature vectors. Using the feature vectors of drugs and diseases, we can easily measure their similarities and therefore identify effective drugs for a given disease. One challenge for the graph embedding method is scalability. Real-world (knowledge) graphs are usually large. The number of entities in a medical knowledge graph could be as many as several million. Existing machine learning systems such as Tensor Flow and PyTorch is mainly designed for data with regular structures but not for large-scale graphs. Therefore, several systems that are specifically designed for learning representations of largescale graphs have been developed. For example, Zhu and colleagues31 developed a high-performance system named GraphVite that could be promising the future drug repurposing because the system can efficiently process tens or even hundreds of millions of nodes [40]. The journey of M.L. Models and AI. in the treatment of patient shown in Figure 6. 


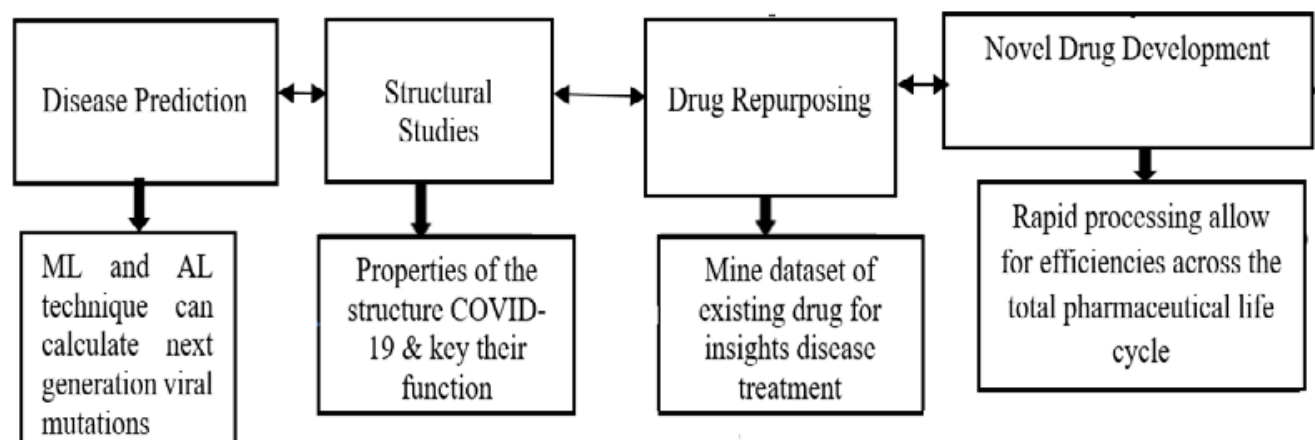

Fig. 6 Journey of M.L. Models and AI. in the treatment of Patient [41]

Increasing interest exists in developing graph representation learning techniques for drug repurposing. Sosa and colleagues constructed a medical knowledge graph of drugs, diseases, genes, and proteins from the biomedical literature and used graph embedding techniques for predicting the links between drugs and diseases. A team constructed a comprehensive COVID-19 knowledge graph (termed CoVKGE) that included 15 million edges across 39 types of relationships connecting drugs, diseases, proteins, genes, pathways, and expressions of genes and proteins35 from a large scientific corpus of 24 million Pub Med publications.
Using Amazon Web Services' computing resources and graph representation, learning techniques, the team identified 41 repurposed drug candidates (including dexamethasone and melatonin) for COVID-19 treatment. To achieve a high predictive performance, the construction of a high-quality medical knowledge graph is essential, which itself is a promising direction for future research. The schematic representation of application of A.l. models Biopharma Chain in Machine Learning Models \& journey for detection to cure with the help of A.l. Model are illustrated in Fig.7 (a, b) respectively.

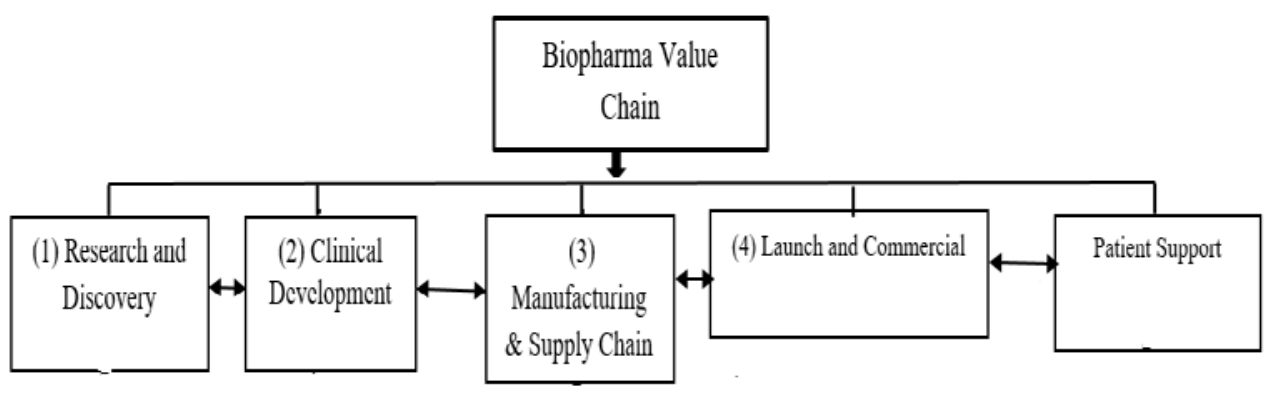

Fig. 7 (a). Uses of A.l. models Biopharma Chain in Machine Learning Models [42].

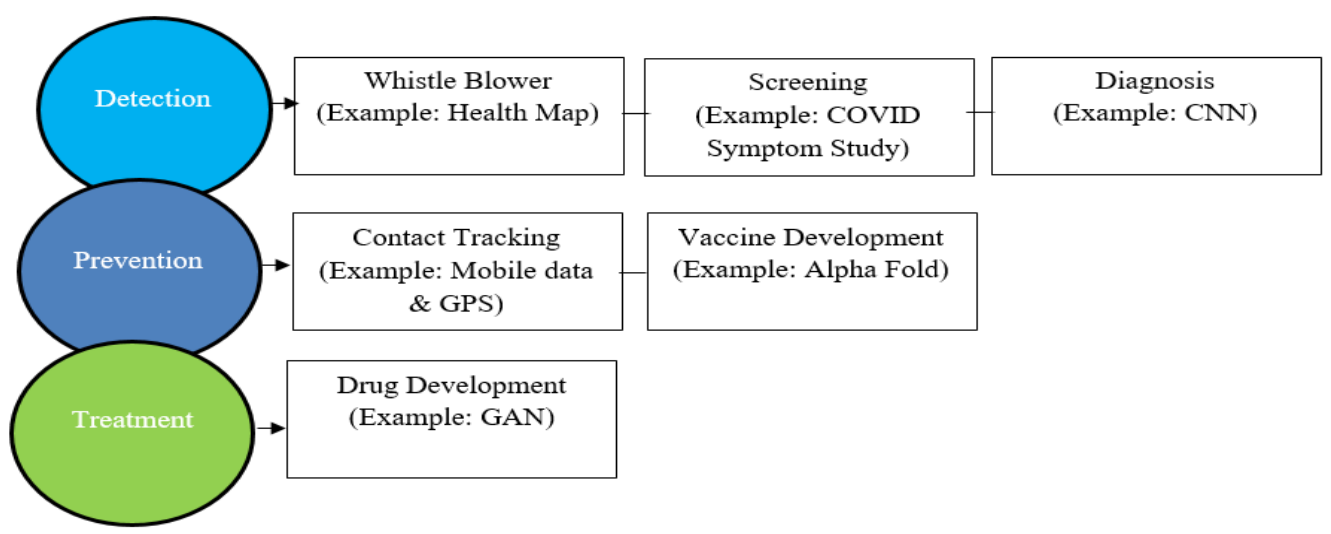

Fig. 7 (b) Journey for Detection to cure with the help of Al Model [43]

\section{APPLICATION DOMAIN}

The artificial neural networks tool is a major tool for solving several problems (machine translation, decisionmaking, visualization, clustering, etc.),
Owing to this it is highly suitable in several distinct areas. The year wise anticipated progress of artificial intelligence is depicted in Figure 8. 


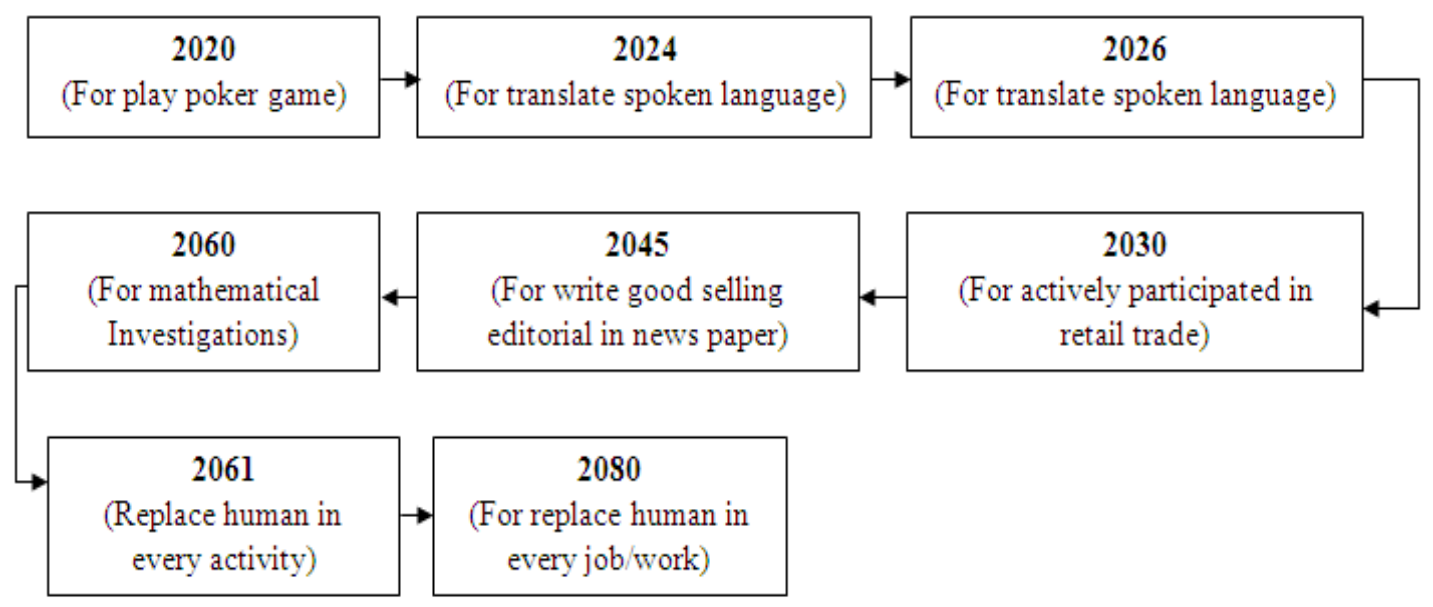

Fig. 8 Anticipated progress of $\mathrm{Al}[44]$

AI enhances lives of man beings by giving personal care of handicap people, aged people, communicating multilingual while not knowing the language, provide help in translating, etc. It has a wide scope in different sectors that include, satellite control, neuromorphic computing, crowd sourcing \& human computation, marketing, military work control, shipping, computer vision, plant layout, nuclear management, education, reinforcement learning, machine learning, deep learning, social media, agriculture, Ecommerce, surveillance etc. In addition, its area of applications are not limited, currently there are major areas where $\mathrm{Al}$ is most widely used are illustrated in Figure 9.



Fig. $9 \mathrm{Al}$ applications in distinct sectors [44]

\section{CONCLUSION}

Artificial Intelligence plays a significant role in the various fields. However, less literature is available for health care, education, social services and fashion sector, etc. The various researchers have only mentioned the technological aspect of artificial intelligence applications. However, there is a need to explore the public administration model for the governance of the administrative state. Presently, Al solves many problems in distinct sectors. It works in a proficient way to mimic like human intelligence and also provide a role in understanding and suggesting the development of a vaccine for Corona virus. Since, the healthcare sectors are in an urgent requirement for decision-making techniques to handle this dangerous virus and help them in getting proper suggestions in real-time to avoid its spread. In addition, artificial intelligence will create luxury of new data science, machine learning, \& IT job positions to promote as well as maintain the systems/ software that will be running those artificial algorithms and enhance the quality of life of mankind. Hence, the result of this technology is utilized for the purpose of screening, prediction, analyzing and tracking of current as well as future patients.

\section{REFERENCES}

[1] S. Mohapatra, "Critical review of literature and development of a framework for application of artificial intelligence in business,” Int. J. Enterprise Network Management, Vol. 10, No. 2, pp.176-185, 2019.

[2] D. D. Luxton, “An introduction to artificial intelligence in behavioural and mental health care,” Academic Press, pp.1-26, 2016. https://doi.org/10.1016/B978-0-12-420248-1.00001-5.

[3] S. Huang, J. Yang, S. Fong and Q. Zhao, "Artificial intelligence in the diagnosis of COVID-19: challenges and perspectives," Int. J. BiolSci , Vol.17, No.1, pp.1581-1587, 2021. doi:10.7150/ijbs.58855.

[4] D.G. Harkut and K. Kasat, “Artifical intelligence- challenges and applications,” pp. 1-5, 2019. DOI: 10.5772/intechopen.84624.

[5] D. Zeng, Z. Cao and D. B. Neill, "Artificial intelligence-enabled public health surveillance-from local detection to global epidemic monitoring and control," Artificial Intelligence in Medicine, Academic Press, 437-453, 2021. 
[6] X. Mei, HC. Lee, and Ky. Diao, et al., "Artificial intelligenceenabled rapid diagnosis of patients with COVID-19," Nat Med., Vol.26, pp. 1224-1228, 2020.

[7] Z. H. Khan., A. Siddique and C. W. Lee, "Robotics utilization for healthcare digitization in global COVID-19 management," International Journal of Environmental Research and Public Health, Vol.17, No. 11, pp.1-21, 2020.

[8] R. Vaishya, M. avoid, I.H. Khan and A. Haleem, "Artificial Intelligence (AI) applications for COVID-19 pandemic," Diabetes and metabolic syndrome, Vol.14, No.4, pp.337-339, 2020.

[9] G. J. Hwang, and Y. F. Tu, "Roles and research trends of artificial intelligence in mathematics education: a bibliometric mapping analysis and systematic review," Mathematics, Vol.9, 584, pp.2-10, 2021. DOI: $10.3390 /$ math9060584

[10] J. Jung, M. Maeda, A. Chang, M. Bhandari, A. Ashapure and J. Landivar-Bowles, "The potential of remote sensing and artificial intelligence as tools to improve the resilience of agriculture production systems," Current Opinion in Biotechnology, Vol.70, pp 15-22, 2021. doi:10.1016/j.copbio.2020.09.003

[11] B. Alhayani, H. J. Mohammed, I. Z. Chaloob, J.S. Ahmed, "Effectiveness of artificial intelligence techniques against cyber security risks apply of IT industry," Materials Today: Proceedings, Vol.30, No.40,pp. 1-6, 2021. DOI:10.1016/j.matpr.2021.02.531

[12] D. Falk, "How artificial intelligence is changing science," 2019.Available:https://www.marketingaiinstitute.com/blog/how-touse-artificial-intelligence-for-analytics. [Accessed 2019].

[13] S. Kumar, R. Cherukuri, "A survey on artificial intelligence techniques in power station," IJIRSET, Vol. 6, No.1, pp. 852-861, 2017.

[14] K. Frankish and W. M. Ramsey, "The Cambridge Handbook of Artificial Intelligence,” Cambridge University Press, 2014.

[15] De. Dombal, F.T. Leaper and D.J. Staniland, et al., "Computer-aided diagnosis of acute abdominal pain,”Br. Med. J., Vol.1, 2 No. 5804, pp. 9-13, 1972. doi: 10.1136/bmj.2.5804.9

[16] S. Kumar, M. Kumar and A. Handa, "Erosion corrosion behaviour and mechanical property of wire arc sprayed $\mathrm{Ni}-\mathrm{Cr}$ and $\mathrm{Ni}-\mathrm{Al}$ coating on boiler steels in actual boiler environment," Material at high temperature, Vol. 37, No.6, pp. 1-15, 2020.

[17] S. Kumar, M. Kumar and A. Handa, "Comparative study of high temperature oxidation behavior of wire arc sprayed $\mathrm{Ni}-\mathrm{Cr}$ and $\mathrm{Ni}-\mathrm{Al}$ coatings," Engineering Failure Analysis, Vol. 106, pp. 104173 104189, 2019.

[18] M. Kumar, S. Kant, and S. Kumar, S., "Corrosion behavior of wire arc sprayed Ni-based coatings in extreme environment”, Materials Research Express, Vol. 6, pp. 106427, 2019.

[19] T.S. Bedi, S. Kumar and R. Kumar, "Corrosion performance of hydroxyapatite and hydroxyapatite/titanium bond coating for biomedical applications," Materials Research Express, Vol.7, pp.116, 2019.

[20] S. Kumar, M. Kumar and S. Handa, "High temperature oxidation and erosion-corrosion behaviour of wire arc sprayed $\mathrm{Ni}-\mathrm{Cr}$ coating on boiler steel," Material Research Express, Vol. 6, No. 12, pp. 1-33, 2019. https://doi.org/10.1088/2053-1591/ab5fae

[21] G. Singh, S. Kumar, and R. Kumar, "Comparative study of hot corrosion behaviour of thermal sprayed alumina and titanium oxide reinforced alumina coatings on boiler steel," Materials Research Express, Vol.7, No2, pp.1-12, 2020.

[22] S. Kumar, M. Kumar and A. Handa, "Combating hot corrosion of boiler tubes- a study,” Journal of Engineering Failure Analysis, Vol. 94, pp. 379-395, 2018.

[23] S. Kumar, M. Kumar and N. Jindal, "Overview of Cold Spray Coatings Applications and Comparisons: A Critical Review”, World Journal of Engineering, Vol.17, No.1, pp. 27-51, 2020. DOI: 10.1108/WJE-01-2019-0021.

[24] V. Sharma, S. Kumar, M. Kumar, D. Deepak, "High Temperature oxidation performance of $\mathrm{Ni}-\mathrm{Cr}-\mathrm{Ti}$ and $\mathrm{Ni}-5 \mathrm{Al}$ coatings", Material Today Proceeding, ICFMST-2019, International Conference at Chandigarh University, Vol. 26, No. 1, pp.1-10, 2019. DOI:10.1016/j.matpr.2019.11.048.

[25] S. Kumar, R. Kumar, S. Singh, et al., "The role of thermal spray coating to combat hot corrosion of boiler tubes: a study," Journal of Xidian University, Vol.14, No.5, pp.229-239, 2020.
[26] H. Singh, S. Kumar and R. Kumar, "Overview of corrosion and its control: a critical review", Proceedings on Engineering Sciences", Vol.3, No.1, pp. 42-49, 2021. DOI: 10.24874/PES03.01.002.

[27] R. Kumar and S. Kumar, "Comparative parabolic rate constant and coating properties of nickel, cobalt, iron and metal oxide based coating: a review," I-Manager's Journal on Material Science, Vol. 6, No. 1, pp. 45-56, 2018.

[28] R. Kumar, R. Singh and S. Kumar, "Erosion and hot corrosion phenomena in thermal power plants and their preventive methods: a study,” Asian Journal of Mechanical Engineering, Vol. 7, No. 1, pp. 38-45, 2018.

[29] R. Kumar and S. Kumar, "Thermal spray coating process: a study," International Journal of Engineering Science and Research Technology, Vol. 7, No. 3, pp. 610-617, 2018.

[30] S. Kumar and R. Cherukuri, "A survey on artificial intelligence techniques in power station,” IJIRSET, Vol. 6, No. 1, pp. 852-861, 2017.

[31] M. R. Davahli, W. Karwowski and K. Fiok et al., "Controlling safety of artificial intelligence-based systems in healthcare,” Symmetry, Vol. 13, No. 1, pp.13-25, 2021.

[32] Sidey-Gibbons, J., Sidey-Gibbons, C. "Machine learning in medicine: a practical introduction,” BMC Med Res Methodol., Vol. 19, No. 64, 2019. https://doi.org/10.1186/s12874-019-0681-4.

[33] H. Singh, S. Kumar and R. Kumar, "Impact of COVID-19 on economy: a critical review,” I-Manager's Journal on Humanities and Social Science, Vol. 2, 2021. DOI: https://doi.org/10.1177/0972262 921989126.

[34] Ian. A. Scott and E. W. Coiera, "Can AI help in the fight against COVID-19,” Medical Journal of Australia, Vol. 213, No.10, pp.439441, 2020. https://doi.org/10.5694/mja2.50821.

[35] J. A. Bullock, G. D. Haddow and D. P. Coppola, "Mitigation, Prevention, and Preparedness," Introduction to Homeland Security, pp. 435-494, 2013. https://doi.org/ 10.1016/B978-0-12-4158023.00010-5

[36] R. Vaishya, M. Javaid, I.H. Khan and A. Haleem, "Artificial Intelligence (AI) applications for COVID-19 pandemic,” Diabetes \& metabolic syndrome, Vol. 14, No. 4, pp. 337-339, 2020.

[37] J. DeCapua, “WHO: Up to 500,000 Spinal Injuries Annually,”2013. [Online]. Available: https://www.voanews.com/science-health/who500000-spinal-injuries-annually. [Accessed 2013].

[38] J. Y. Chen and M. D. T. M. Sippel Schmid et al., "Enabling the NextGeneration Radiology Report: Description of Two New System Standards, $R G, \quad$ Vol. $\quad 37, \quad$ No.7, $\quad$ pp.2106-2112, 2017. https://doi.org/10.1148/rg.2017160106

[39] O. O. Donnell, "Access to health care in developing countries: breaking down demand side barriers," CadSaude Publica, Vol. 23, No.12, pp. 2820-2834, 2007. doi:10.1590/s0102-311x20070 01200003

[40] Y. Zhou, F. Wang, J. Tang, R. Nussinov and F. Cheng, "Artificial intelligence in COVID-19 drug repurposing," The LANCET Digital Health, Vol. 18, pp. 1-10, 2020. DOI: https://doi.org/10.1016/ S2589-7500(20)30192-8

[41] Z. Ahmed, K. Mohamed, S. Zeeshan and X. Qi Dong, "Artificial intelligence with multi-functional machine learning platform development for better healthcare and precision medicine," Journal of Biological Database, Vol. 2020, pp.1-12, 2020.https://doi.org/ 10.1093/database/baaa010

[42] M. Steedman, K. Tayler and F. Properzi, "The rise of artificial intelligence across biopharma,” 2019. [Online]. Available: https://www2.deloitte.com/us/en/insights/industry/life-sciences/riseof-artificial-intelligence-in-biopharma-industry.html. [Accessed 2019].

[43] S. Weib, "Journey for Detection to cure with the help of Al Model," 2020. [Online]. Available: https://www.zuehlke.com/en/insights/ how-ai-is-already-transforming-the-patient-journey. [Accessed 2020].

[44] D. G. Harkut, K. Kasat and V. D. Harkut, "Introductory Chapter: Artificial Intelligence - Challenges and Applications,” Intech Open, pp. 1-5. DOI:10.5772/intechopen.84624. 